\title{
Rassismuskritik als Identitätspolitik?
}

\section{Anfragen an ein allzu einfaches Weltbild und seine Kritik}

Die Frage nach den Grundlagen und Perspektiven einer angemessenen Rassismuskritik führt anhaltend zu zugespitzten Kontroversen um identitätspolitische Positionen. In diesen Kontroversen wird wiederkehrend mit Vereinfachungen und Zuspitzungen argumentiert, die der komplexen Gemengelege der Strukturen und Praktiken, die die gegenwärtigen Ungleichheitsverhältnisse und Diskriminierungsformen kennzeichnet, nicht gerecht werden.

W issenschaftliche Analysen sind deshalb darauf verwiesen, sich nicht der einen oder anderen Seite von Befürworter_innen und Gegner_innen von Identitätspolitik zuzuordnen, sondern zu beiden Seiten hinreichende Distanz einzunehmen, um Verkürzungen und Blindstellen kritisch analysieren zu können. „Genau hinsehen, geduldig nachdenken, sich nicht dumm machen lassen“ (Steinert 1998, S. 67), diese Aufgabenbestimmung wissenschaftlicher Arbeit ist auch im Fall des Streits über identitätspolitische Positionen in der Diskriminierungs- und Rassismuskritik nicht hintergehbar.

\section{Rassismuskritik, auch in der Mitte der Gesellschaft}

Dass es erforderlich ist, Rassismus wissenschaftlich zu analysieren und praktisch dagegen vorzugehen (politisch, rechtlich, pädagogisch, in Schulen und Hochschulen, in der Sozialen Arbeit, in den Massenmedien, usw.) ist inzwischen - jenseits rechtsextremer und rechtspo-
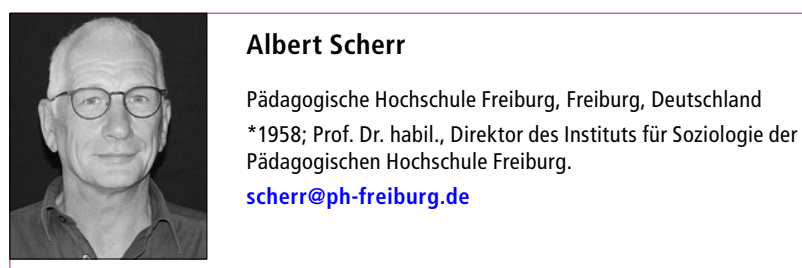

Pädagogische Hochschule Freiburg, Freiburg, Deutschland *1958; Prof. Dr. habil., Direktor des Instituts für Soziologie der Pädagogischen Hochschule Freiburg.

scherr@ph-freiburg.de

Zusammenfassung Über die wissenschaftliche Tragfähigkeit und die gesellschaftlichen Konsequenzen identitätspolitischer Positionen, wie sie nicht zuletzt in der Rassismuskritik einflussreich sind, hat sich eine heftige Kontroverse entwickelt. Der Beitrag zeigt Erfordernisse und Perspektiven einer differenzierten Auseinandersetzung mit unterschiedlichen Spielarten von Identitätspolitik und ihrer Kritik auf.

Schlüsselwörter Kollektive Identität, Universalismus, Diskriminierung, Rassismuskritik pulistischer Strömungen - unstrittig. Und es sind längst auch nicht mehr nur antirassistische Aktivist_innen, sondern auch von der Bundes- und Landesregierungen und finanzstarken Stiftungen geförderte Programme und Projekte, die sich explizit gegen Rassismus wenden. So nimmt etwa die deutsche Bundesregierung in ihrer „Strategie der Bundesregierung zur Extremismusprävention und Demokratieförderung “ (Bundesregierung 2016, S. 7) in Anspruch, dass der „Schutz aller Menschen vor Rassismus und Diskriminierung .... für die Bundesregierung ein Ziel von herausragender Bedeutung " ist.

Auch der Deutsche Fußballbund und die Deutsche Fußballliga sehen sich veranlasst, den „Kampf gegen Rassismus “ zu ihrem Ziel zu erklären und die Internationalen Wochen gegen Rassismus zu unterstützen. ${ }^{1}$ In den USA haben Großkonzerne wie Youtube, Warner und Sony in Reaktion auf die Black-Live-Matters-Bewegung ca. zwei Milliarden Dollar für Initiativen und Organisationen in Aussicht gestellt, die sich gegen Rassismus einsetzen (Mayer 2021, S. 238). Mayer (2021, S. 241) stellt diesbezüglich fest: „Inzwischen sehen auch führende Konzerne ebenso wie die Führung der demokratischen Partei, dass die Positionen innerhalb der Klassenspaltung durchaus fairer entlang ethnischer und identitärer Merkmale zu verteilen seien - solange die Klassenspaltung selbst nicht angetastet wird." Darin zeigt sich exemplarisch, dass Rassismuskritik gegenwärtig nicht mehr als eine radikal-oppositionelle Gesellschaftskritik verstanden werden kann, sondern politisch mit dem Selbstverständnis bürgerlich-demokratischer Gesellschaften und ökonomisch mit der Tendenz zur Herausbildung eines globalisierten und post-rassistischen $\mathrm{Ka}$ pitalismus übereinstimmt, in dem historisch gewordene Verkoppelungen von Ungleichheiten zwischen sozialen Klassen mit rassistischen Hierarchien schrittweise auf- 
gelöst werden (Scherr 2017). ${ }^{2}$ Es ist folglich wenig verwunderlich, sondern als Ausdruck einer veränderten gesellschaftlichen Position von Rassismuskritik zu interpretieren, dass Rassismusforschung in Deutschland seitens der Bundesregierung inzwischen mit erheblichen Mitteln gefördert wird. ${ }^{3}$

Auch Formen einer identitätspolitischen, an der Perspektive der „Kritischen Weißseinsforschung“ ausgerichteten Perspektive der Rassismuskritik ${ }^{4}$ finden inzwischen nicht mehr nur in antirassistischen linken Milieus Resonanz: „Hört auf zu jammern, alte weiße Männer!“ (Raether 2018) - unter dieser Überschrift wurde im Magazin der Süddeutschen Zeitung, also in einer Publikation, die sich vor allem an die bildungsbürgerlichen Schichten der Mehrheitsgesellschaft wendet, dargelegt, dass und warum die so Adressierten sich ihrer Privilegien bewusst werden und Abwehrhaltungen gegen eine Auseinandersetzung mit dem Privileg des Weißseins überwinden sollen. Zentrale Referenz dieses Texts ist die Studie der us-amerikanischen Soziologin Robin DiAngelo, „White Fragility“ (DiAngelo 2018a), die in den USA zum New-York-Times-Bestseller avanciert und dann 2020 in einer deutschen Übersetzung unter den Titel „Wir müssen über Rassismus sprechen“ erschienen ist. Von der sich selbst als Weiße bezeichnenden Autorin wird zu kritischer Selbstreflexion aufgefordert: „Wir müssen lernen, die weiße Überlegenheit, die wir alle internalisiert haben, zu reflektieren." (ebd.). Dies wird mit einem Verständnis von Rassismus nicht nur als strukturelles Merkmal gegenwärtiger Gesellschaften verbunden, sondern auch als eine ,internalisierte Haltung, die in jeder und jedem Weißen steckt“" (DiAngelo 2018b). Ebenfalls den Status eines Bestsellers hat in den USA das Buch „Why I'm No Longer Talking To White People About Race" von Reni Eddo-Lodge erreicht, das 2019 in deutscher Übersetzung veröffentlicht wurde und als Sonderausgabe der Bundeszentrale für politische Bildung verbreitet wird. Angeklagt wird dort die angenommene „weiße Verweigerung“ (EddoLodge 2019, S. 11), sich mit Rassismus auseinanderzusetzen und behauptet, dass diese eine Folge eines fehlenden Interessen von Weißen sei, ihre eigene privilegierte Position zu hinterfragen.

\section{Kollektive Identitäten und Politik}

Solche generalisierenden Behauptungen über „die Weißen“ provozieren Widerspruch auch von Autor_innen, die für sich selbst eine rassismuskritische Position als Ausdruck demokratischer und menschenrechtlicher Überzeugungen beanspruchen und davon ausgehen, dass diese nicht von der eigenen Zugehörigkeit zu einer Minderheit oder Mehrheitsgesellschaft abhängig ist. „Der Kampf gegen Rassismus ist eine demokratische Pflicht“ und nicht „Herkunft oder Hautfarbe entscheiden dabei, sondern Haltung “, wendet der ehemalige Bundespräsident Joachim Gauck (2021, S. 55) ein, und diese Haltung sei „unabhängig von der Hautfarbe“. Er widerspricht zudem auch der für die identitätspolitische Rassismuskritik zentralen Behauptung, dass Weiße schon aufgrund ihrer Hautfarbe privilegiert seien: „Für die übergroße Mehrheit auch der weißen Menschen ist die Geschichte eine Geschichte von Inferiorität, Ohnmacht und eingeschränkten Lebensmöglichkeiten. Für eine Minderheit ist sie Suprematie und Herrschaftsgeschichte.“ (Gauck 2021, S. 56).

Dieses Verständnis von Rassismuskritik reklamiert ein gemeinsames Interesse Aller an der Überwindung jedweder Formen von Diskriminierung und steht damit in einem Gegensatz zu identitätspolitischen Positionen, die annehmen, dass niemand der Überformung eigener Erfahrungen durch rassistische Privilegierung und Benachteiligung entkommen kann: „Das Konzept der Farbenblindheit ist eine infantile, schlecht durchdachte Analyse des Rassismus. Sie beginnt und endet mit ,eine Person wegen ihrer Hautfarbe zu diskriminieren ist schlecht', ohne die unterschiedlichen Arten und Weisen anzuerkennen, auf die sich struktureller Macht in einem solchen Austausch manifestiert. (...) Farbenblindheit leugnet strukturellen Rassismus und die Geschichte weißer Dominanz. Und sich selbst immer wieder einzureden und schlimmer noch, unseren Kindern einzureden -, dass wir alle gleich sind, ist eine irreführende, wenn auch wohlmeinende Lüge. “ (Eddo-Lange 2019, S. 93f.). In dieser Formulierung wird zunächst eine zweifellos plausible Sichtweise deutlich, die davon ausgeht, dass das Verhältnis von People of Color und Weißen auch gegenwärtig noch vielfach durch Macht- und Ungleichheitsverhältnisse überformt ist und es naiv wäre, die Bedeutung unterschiedlicher Erfahrungen zu verleugnen. Problematisch ist die Kritik der Forderung nach Farbenblindheit jedoch dann, wenn dies zu einer Position führt, in der Rassismuskritik als Auseinandersetzung zwischen zwei eindeutig unterscheidbaren und in sich homogenen Kollektiven konzipiert wird, in der Schwarze bzw. People of Color als Opfer von Rassismus in eine Auseinandersetzung mit Weißen als Profiteuren von Rassismus eintreten. Denn damit wird vernachlässigt, dass sozioökonomische Ungleichheiten, Geschlechterverhältnisse, Generationsverhältnisse, jeweilige Situierungen in historischen und nationalgesellschaftlichen Verhältnissen sowie unterschiedlicher Formen von Rassismen nicht sinnvoll mit einem vereinfachenden binären SchwarzWeiß-Schema verstanden werden können. Problematisch ist auch die unterschiedliche identitätspolitische 
Konzepte kennzeichnende Perspektive, die davon ausgeht, dass gesellschaftliche Strukturen eine direkte Entsprechung in den Erfahrungen, Lebenslagen und im Selbstverständnis von klar voneinander abgrenzbaren Kollektiven haben. ${ }^{5}$ Identitätspolitische Positionen basieren jedoch auf der Annahme, dass kollektive Identitäten ein Ergebnis spezifischer historischer und aktueller Erfahrungen sind sowie eine unhintergehbare Grundlage politischer Überzeugungen und organisierter Interessenvertretung sein sollen. ${ }^{6}$ Identitätspolitik grenzt sich damit ebenso gegen ein bürgerlich-individualistisches Verständnis von Politik ab, das davon ausgeht, dass freie und gleiche Bürger_innen die Subjekte politischen Handelns sind, wie gegen ein Politikverständnis, das Politik als Interessenvertretung sozialer Klassen fasst.

\section{Bürgerlich-liberale und linke Kritik der Identitäts- politik}

Diese zweifache Abgrenzung ist jedoch asymmetrisch: Zwischen einem bürgerlich-individualistischen Politikverständnis und identitätspolitischen Positionen besteht ein grundlegender Gegensatz, da Gesellschaft hier als ein politischer Zusammenhang von Individuen - also gerade nicht von Kollektiven - gedacht wird, die sich wechselseitig als mündige Subjekte anerkennen sollen. Dementsprechend provoziert Identitätspolitik den bürgerlich-liberalen Einwand, die zentrale Bedeutung individueller Rechte zu verkennen und deshalb mit demokratischen und menschenrechtlichen Prinzipien nicht vereinbar zu sein (s. Pfahl-Traughber 2020 und 2021). Dagegen ist die Vorstellung, dass Klassen, die eine kollektive Identität (Klassenbewusstsein) ausbilden können und Kollektivsubjekte politischen Handels sind, in linken Theorien und Politikkonzepten verankert. Die Vorstellung, dass Menschen nicht nur Individuen, sondern auch - und ggf. vor allem - Angehörige eines Kollektivsubjekts sind, unterscheidet bestimmte Spielarten marxistischer Klassentheorien also nicht von ethnischen und nationalistischen Identitätskonstruktionen, sondern allein die inhaltliche Bestimmung des Kollektivsubjekts, das als Subjekt politischen Handelns angenommen wird. Dementsprechend wendet sich die linke Kritik der Identitätspolitik nicht zentral gegen deren Kollektivismus identitätspolitischer Konzepte, sondern gegen die Vernachlässigung der Bedeutung von Klassenlagen und klassenspezifischer Interessen. Sie verbindet dies mit dem ideologiekritischen Verdacht, dass es sich um die Artikulation milieuspezifischer Präferenzen von ökonomisch Privilegierten handelt (s. etwa Michels 2006; Wagenknecht 2021).

Zudem ist zu berücksichtigen, dass (neo-)marxistische Theorien in der Tradition des westlichen humanistischen Marxismus und der Frankfurter Schule - im
Gegensatz zu ihren leninistischen, stalinistischen und maoistischen Varianten - in ihrer utopischen Zielperspektive auf die Überwindung von Klassenverhältnissen in einer zukünftigen Gesellschaft zielen, die als eine freie Assoziation freier und gleicher Individuen gedacht wird (s. dazu Vranicki 1974). Sie lehnen also das bürgerlichliberale Ideal der individuellen Freiheit und Selbstbestimmung sowie menschenrechtliche Werte keineswegs ab. Dagegen werden Zugehörigkeiten zu Völkern und Nationen mit einer kulturell, ethnisch oder rassistisch gedachten Identität in der Programmatik der alten und neuen Rechten als positive und zu verteidigende Tatsachen behauptet und mit Ideologemen verbunden, die von Individuen Ein- und Unterordnung fordern. In rechtpopulistischen und rechtsextremen Varianten wird dies mit der auf Carl Schmitt zurückgehenden anti-pluralistischen und anti-demokratischen Idee einer identitären Demokratie verbunden, die durch eine rassistisch oder ethnisch gefasste Identität von Regierten und Regierenden gekennzeichnet ist (Niethammer 2000, S. $120 \mathrm{ff}$. und $482 \mathrm{ff}$.).

Vor diesem Hintergrund kann festgestellt werden: Identitätspolitische Konzepte sind erstens im Hinblick darauf zu unterscheiden, wer in ihren Theorien und Ideologien als Kollektivsubjekt politischen Handelns angenommen wird. Zweitens stellt es eine substanzielle Differenz dar, wie das Verhältnis der Ein- und Unterordnung in ein jeweiliges Kollektiv zur Autonomie und Freiheit der Individuen gedacht wird und ob als Ziel politischer Gesellschaftsgestaltung die konservative Verteidigung bzw. reaktionäre Wiederherstellung, oder aber die progressive Überwindung einer gesellschaftlichen Ordnung gedacht wird, in der kollektive Zugehörigkeiten die individuelle Freiheit und Selbstbestimmung einschränken. Eine Gemeinsamkeit zwischen bürgerlich-liberalen und emanzipatorisch-linken Politikkonzepten besteht darauf bezogen darin, dass sie im Grundsatz eine Gesellschaft anstreben, in der die Freiheit der Individuen als zentraler Wert betrachtet wird. Das unterscheidet sich von solchen Politikkonzepten, die kollektive Identitäten und Interessen als vorrangig betrachten und die Würde des Individuums und individuelle Rechte demgegenüber als nachrangig oder vernachlässigbar einordnen. Mit der Unterscheidung demokratische Mitte vs. linker und rechter Extremismus sind diese Unterschiede nicht zu fassen, und auch nicht mit einem einfachen Links-Rechts-Schema. Folglich kann Identitätspolitik nicht generell als progressiv oder reaktionär bzw. als links oder rechts gelten, und sie ist auch nicht notwendig Bestandteil autoritärer oder demokratischer Gesellschaftsmodelle.

In der Konsequenz heißt das: Der Verdacht, dass linke und rechte Identitätspolitik eine „gefährliche Nähe“ 
aufweisen könnte (Pfahl-Traughber 2021), ist nicht von vornherein obsolet. Er ist aber zweifellos nicht angemessen mit den Mitteln einer solchen Extremismustheorie zu fassen, die linke Positionen unter den Generalverdacht stellt, individuelle Freiheitsrechte und demokratische Prinzipien abzulehnen. ${ }^{7}$ Darüber hinaus ist zu berücksichtigen, dass auch zwischen politischen Beanspruchungen nationaler Identität seitens demokratischer Parteien und rechtsextremen Konzepten identitärer Demokratie unscharfe Grenzen bestehen. Insofern kann es nicht um eine generelle Befürwortung oder Ablehnung von Identitätspolitik, sondern nur um eine differenzierte Auseinandersetzung mit ihren unterschiedlichen Ausprägungen gehen.

\section{Akzeptierte und strittige Formen von Identitäts- politik}

In minderheitenpolitischen bzw. antirassistischen Spielarten von Identitätspolitik wird - in deutlichem Gegensatz zu nationalistischer Identitätspolitik - nicht die vermeintliche Einheit von Volk und Nation, sondern Differenz betont: Kollektive Identitäten werden hier als ein Unterscheidungsmerkmal zur sog. Mehrheitsgesellschaft beansprucht, das auf unterschiedliche Formen von Ausbeutung, Ausgrenzung und Unterdrückung bis hin zu physischer Vernichtung und Völkermord verweist. In der Folge zielen minderheitenpolitische bzw. antirassistische Identitätspolitiken auf Anerkennung der Ungerechtigkeiten und des Leidens, auf materielle und symbolische Entschädigungen sowie auf die konsequente Überwindung gegenwärtiger Formen von Diskriminierung.

In prominenten Fällen - so in Deutschland im Fall der jüdischen Minderheit und inzwischen auch der Sinti und Roma - ist die Legitimität einer solchen Identitätspolitik prinzipiell unstrittig, ${ }^{8}$ ebenso wie eine Politik im Namen christlicher religiöser Identitäten, z. B. als politische Vertretung des Katholizismus und des Protestantismus. Dies wird auch auf der Seite derjenigen nicht in Frage gestellt, die dezidiert Position gegen eine antirassistische Identitätspolitik beziehen. Darüber hinaus kann mit Robert Misk (2019, S. 102ff.) festgestellt werden, dass es keine guten Gründe gibt, Minderheiten das Recht zu bestreiten, ihre spezifischen Erfahrungen mit Benachteiligung, Ausgrenzung und Unterdrückung zu thematisieren und politisch zu artikulieren. Zweifellos ist jede Identitätspolitik im Namen von Minderheiten zwar schon deshalb problematisch, weil die Fragen, was die je eigene kollektive Identität kennzeichnet und welche politische Folgerung aus der eigenen Geschichte und der gegenwärtigen Situation zu ziehen ist, innerhalb von Minderheiten ebenso vielfach strittig sind, wie die Frage, wer mandatiert ist, die jeweilige Minderheit legitim zu repräsentieren bzw. in ihrem Namen zu sprechen. Diese Problematiken sind aber kein prinzipieller Einwand gegen Positionen, die geltend machen, dass diskriminierte Minderheiten ein Recht haben sollen, historische und gegenwärtige Formen von Diskriminierung politisch zu thematisieren. Gibt es gleichwohl gute Gründe, gegenwärtige Formen antirassistischer Identitätspolitik zu kritisieren?

In seiner klugen Kommentierung der anhaltenden Debatte wendet Robert Misk (2019, S. 106) gegen die linke Kritik an Identitätspolitik ein, dass es „keine besonders schwierige Übung “ sei, ,gleichzeitig sowohl die generellen sozialen Ungleichheiten als auch die speziellen Diskriminierungen einzelner Minderheiten zu kritisieren“, also das eine nicht gegen das andere auszuspielen. Das ist auf den ersten Blick zweifellos einleuchtend, blendet aber aus, dass die Thematisierung sozioökonomischer Ungleichheiten in einem erheblichen Spannungsverhältnis zu Versuchen steht, eine widerspruchsfreie kollektive Identität von Minderheiten anzunehmen: Denn die Frage, in welchem Sinn und mit welcher politischen Relevanz noch von einer kollektiven Identität gesprochen werden kann, wenn zugleich zu berücksichtigen ist, dass auf die Hautfarbe und andere körperliche Merkmale bezogene bzw. durch kulturrassistische Zuschreibungen bedingte Rassismuserfahrungen nur eine recht begrenzte Gemeinsamkeit begründen - z. B. zwischen einem/ einer Professor_in mit deutscher Staatsangehörigkeit, einer prekär beschäftigten Putzkraft mit Aufenthaltserlaubnis und abgelehnten Asylbewerber_innen -, ist keineswegs überzeugend mit der Behauptung zu beantworten, dass Rassismuserfahrungen für das individuelle Selbstverständnis dominant sind bzw. sein sollen.

Der Diskurs des identitätspolitischen Rassismus hat auf diese Problematik mit der Forderung reagiert, Schwarzsein und Weißsein nicht biologistisch, sondern als Positionen innerhalb rassifizierter Machtverhältnisse zu verstehen (s. dazu Kerner 2013). Diese wissenschaftliche Position ist inzwischen in den politischen Diskurs eingegangen, was exemplarisch in folgender Formulierung von Amnesty International deutlich wird: „,Weiß‘ und ,Weißsein' bezeichnen ebenso wie ,Schwarzsein' keine biologische Eigenschaft und keine reelle Hautfarbe, sondern eine politische und soziale Konstruktion. Mit Weißsein ist die dominante und privilegierte Position innerhalb des Machtverhältnisses Rassismus gemeint, die sonst zumeist unausgesprochen und unbenannt bleibt. Weißsein umfasst ein unbewusstes Selbst- und Identitätskonzept, das weiße Menschen in ihrer Selbstsicht und ihrem Verhalten prägt und sie an einen privilegierten Platz in der Gesellschaft verweist, was z. B. den Zu- 
gang zu Ressourcen betrifft.“ (Amnesty International 2017).

Dies führt jedoch nicht zu einer überzeugenden Lösung des Problems, in welcher Weise von einer weißen oder schwarzen Identität trotz vielfältiger sonstiger Unterschiede ausgegangen werden kann: Problematisch daran ist nicht nur, dass für Rassismus die Hautfarbe und andere körperliche Merkmale ersichtlich eine zentrale Grundlage von Positionszuweisungen sind. Denn nur deshalb ist die Rede von Weißsein und Schwarzsein ja nicht völlig beliebig und nicht einfach durch die Rede von Privilegierten und Benachteiligten zu ersetzen. Vor allem aber ist damit die Frage nicht beantwortet, welche gesellschaftliche Bedeutung Rassismen für das Selbstverständnis von Individuen haben, die eben nicht als Schwarze, People of Color oder Weiße klassifiziert werden, sondern auch Staatsbürger_innen oder Nicht-Staatsbürger_innen, Unternehmer_innen, Beamt_ innen, Angestellte, Arbeiter_innen oder Arbeitslose, religiös Gläubige oder Atheist_innen usw. sind. Hinzu kommt, dass unterschiedliche Teilgruppen derjenigen, die in dieser Weise als Schwarze oder People of Color adressiert werden, sich nicht selbstverständlich in einem gemeinsamen Erfahrungszusammenhang verorten, sondern ethnische, nationalistische, religiöse und politische - sowie ggf. auch rassistische - Abgrenzungen und Konflikte der Verständigung auf eine geteilte Identität entgegenstehen. Antirassistische Identitätspolitik basiert folglich auf einer durchaus prekären Imagination und Anrufung von Gemeinsamkeiten.

Weiß und Schwarz, Unterdrücker und Unterdrückte? Identitätspolitische Positionen, die von einer einfachen Einteilung in privilegierte Weiße und benachteiligte Schwarze ausgehen, ${ }^{9}$ stellen ersichtlich eine sehr vereinfachende Sicht auf die gesellschaftliche Wirklichkeit dar. ${ }^{10}$ Wenn dies dann noch mit generalisierenden $\mathrm{Zu}$ schreibungen einhergeht, die insbesondere den „Netten Weißen Leuten“ einen „Mangel an Verständnis und Empathie" (Eddo-Lodge 2019, S. 12) unterstellt und dies mit der Konsequenz verbindet, „mit Weißen nicht mehr über die Hautfarbe“ zu sprechen, „außer es lässt sich absolut nicht vermeiden“, ist es wenig verwunderlich, wenn dies zu Abwehrreaktionen führt. Diese können auch, aber keineswegs nur, als eine Verweigerung selbstkritischer Auseinandersetzung von privilegierten Weißen mit ihren Privilegien verstanden werden. Denn es ist zwar nicht sinnvoll zu bestreiten, dass es zweifellos ein „White Privilege“ gibt, das in der „Abwesenheit von negativen Folgen von Rassismus“ (ebd.: S. 97) im eigenen Erfahrungszusammenhang besteht. Das heißt aber nicht, dass als weiß Etikettierte nicht von anderen For- men von Benachteiligung betroffen sein können und sich deshalb mit guten Gründen selbst nicht als gesellschaftlich Privilegierte begreifen und auch nicht als solche adressiert werden möchten. Zudem sind diejenigen, die als Staatsbürger_innen der wohlhabenden und demokratisch verfassten Staaten des globalen Nordens von rassistischer Diskriminierung betroffen sind, als Staatsbürger_innen selbst Privilegierte im Verhältnis zur Mehrzahl der Staatsbürger_innen der Länder des globalen Südens. Der anklagende Ton, mit dem dann als Weiße Wahrgenommene aufgefordert werden, sich mit ihren eigenen rassistischen Privilegierung zu befassen, setzt insofern voraus, dass die Ankläger_innen, vielfach Akademiker_ innen und qua Staatsbürgerschaft Privilegierte, die Thematisierung von Rassismus mit einer Ausblendung ihrer eigenen Privilegien verbinden und die Frage vermeiden, was im Verhältnis von Staatsbürgerschaft, Klassenlage und rassistischer Diskriminierung die folgenreicheren Formen von Privilegierung und Benachteiligung sind.

Die damit skizzierten Schwierigkeiten, zu denen der Versuch führt, die Diskriminierungsrealität in ein Schwarz-Weiß-Schema einzuordnen, werden exemplarisch im folgenden Zitat aus einem Interview mit einem in der pädagogischen und sozialarbeiterischen Diskussion einflussreichen Vertreter der akademischen Rassismuskritik deutlich: „Rassismus ist ein weißes Dominanzsystem, deshalb gibt es beispielsweise keine Deutschenfeindlichkeit. (...) People of Color haben nicht die Macht, Schwarze Menschen vom Bildungsoder Arbeitsmarkt auszuschließen, weiße Menschen haben sie. People of Color können also nur situativ diskriminieren. Rassismus hat immer mit Machtstrukturen und Traditionslinien zu tun.“ (Fereidooni 2020).

Kritisch anzumerken ist dazu erstens, dass hier im ersten Satz Rassismus mit Nationalismus konfundiert wird und Rassismus, global betrachtet, auch nicht durchgängig ein weißes Dominanzsystem war und ist (Dikötter 2018). Zweitens gibt es durchaus Fälle, in denen People of Color die Macht haben, Schwarze auszuschließen, wenn sie z. B. die Position von Professsor_innen oder einer Führungskraft im Management eines Unternehmens einnehmen. Man kann zweifellos kritisieren, dass dies zu selten der Fall ist, aber nicht behaupten, dass dies prinzipiell nie der Fall sein kann, ganz abgesehen davon, dass es in zahlreichen Ländern der Welt sehr mächtige und sehr reiche Schwarze Menschen und People of Color gibt. Dass People of Color nur situativ diskriminieren können, ist drittens eine zweifellos zutreffende Relativierung, aber das Gleiche gilt - jedenfalls in Gesellschaften, die nicht auf rechtlich und politisch verankerten Rassenhierarchien basieren - auch für diejenigen, die als Weiße etikettiert werden. Viertens ist die 
Formulierung, dass Rassismus immer mit Machtstrukturen und Traditionslinien zu tun hat, missverständlich, wenn dabei ausgeblendet wird, dass die Ablehnung von manifestem Rassismus in der Europäischen Union und in Deutschland in das Selbstverständnis von Regierungen und demokratischen Parteien sowie in die Rechtsordnung eingeschrieben ist, es also allzu einfach wäre, Rassismus als ungebrochenes hegemoniales Machtverhältnis zu begreifen. Zudem ist zu berücksichtigen, dass Rassismus auch eine Artikulationsform von Unzufriedenheit bei denjenigen sein kann, die sich - als Benachteiligte innerhalb jeweiliger Macht- und Ungleichheitsverhältnisse - erleben.

\section{Rassismus und Antisemitismus}

Eine weitere substanzielle Schwierigkeit unterkomplexer Varianten von Rassismuskritik resultiert daraus, dass die Problematik des Antisemitismus in einem binären Schwarz-Weiß-Schema nicht sinnvoll unterzubringen ist. Denn ein wichtiges Konstruktionselement des historischen und gegenwärtigen antisemitischen Rassismus ist die Vorstellung, Juden seien eine besonders mächtige und einflussreiche Gruppe. „Die Juden und ihre globale Verschwörung beherrschen die Welt, und es sei Deutschlands Pflicht, sie zu vernichten, bevor sie die Menschheit auf ewig unterwerfen würden “, so fasst Batov (2004, S. 21) treffend ein zentrales Element des nationalsozialistischen Antisemitismus zusammen. Definiert man Rassismus exklusiv als Ideologie im Verhältnis machtvoller Weißer zu machtlosen Schwarzen, dann stellt sich Antisemitismus nicht als eine Form von Rassismus dar. Dies kann zu einer Kritik israelischer Regierungspolitik führen, die von Formen eines sekundären Antisemitismus nur noch schwer zu unterscheiden ist. Dass in einigen Strömungen des linken Diskurses eine Wahrnehmung von israelischen Juden als weiße Kolonisatoren aufscheint, führt bei Mirna Funk (2021, S. 3) deshalb zu folgender polemisch zugespitzter Kritik: „Das Postcolonial-Studies-Halbwissen wurde mit angesagten Social-Media-Begriffen aufgeladen und das Ganze von der woken Gerechtigkeitsbewegung übernommen, deren Ideologie darauf basiert, dass wir in einer binären Welt aus Unterdrückern und Unterdrückten leben. Herausgekommen ist dabei ein fresher und angesagter Antizionismus. (...) Die gegenwärtigen Social-Justice-Bewegungen, die sich selbst, vermutlich fälschlicherweise, als links definieren und fest daran glauben, progressiv zu sein, repräsentieren das Gegenteil von freiheitlichem Denken und Handeln: Sie sind in Teilen autoritär und ideologisch. Sie sind nicht nur der unterkomplexen Idee verfallen, wir lebten in einer dichotomen Welt von Unterdrückern und
Unterdrückten. Sie sitzen auch dem Irrtum auf, es gebe eine absolute Wahrheit. Und sie trügen sie in die Welt.“ Hingewiesen ist damit darauf, dass ein solcher identitätspolitischer Antirassismus, der mit einer grob vereinfachenden binären Weltsicht und der Äquivalenzkette „Herrschende und Beherrschte - Privilegierte und Benachteiligte - Weiße und Schwarze - Gut und Böse “ operiert, keineswegs zufällig nicht zu einer angemessenen Analyse des Nahostkonflikts befähigt. Denn nicht nur in diesem Fall ist das zugrunde liegende Schema zu einfach, um komplexen, in sich widersprüchlichen und dynamischen gesellschaftlichen Verhältnissen gerecht zu werden.

\section{Und wie weiter?}

Es kann in politischen Auseinandersetzungen sowie in der Praxis der schulischen und außerschulischen Bildungsarbeit keineswegs darum gehen, die Legitimität identitätspolitischer Rassismus- und Diskriminierungskritik prinzipiell zu bestreiten. Denn diese ist ein zweifellos berechtigter Ausdruck spezifischer historischer und gegenwärtiger Erfahrungen benachteiligter Minderheiten. Gleichwohl aber ist zu berücksichtigen, dass Annahmen über eindeutig konturierte kollektive Identitäten mit folgenreichen Ausblendungen einhergehen sowie dass eine Kritik, die von der Vorstellung einer durchgängig rassistisch strukturierten Gesellschaft ausgeht, den gegenwärtigen gesellschaftlichen Verhältnissen nicht gerecht wird. Die in politische Diskursen eingeschriebene Tendenz zu Vereinfachungen, Vereindeutigungen und polemischen Zuspitzungen verschafft einem identitätspolitischen Schwarz-Weiß-Schema zweifellos ebenso eine gewisse Attraktivität, wie das Interesse der Medien, die Aufmerksamkeit des Publikums durch die Inszenierung unversöhnlicher Kontroversen zu gewinnen. In der wissenschaftlichen Forschung und der Bildungsarbeit ist es jedoch gerade erforderlich, dies kritisch zu reflektieren, statt simple Vereindeutigungen fortzuschreiben und damit hinter das erreichten Reflexionsniveau rassismuskritischer Theorie und Praxis ${ }^{11}$ zurückzufallen.

Eingegangen. 24. Juni 2021

Angenommen. 23. Juli 2021

Funding. Open Access funding enabled and organized by Projekt DEAL.

Open Access. Dieser Artikel wird unter der Creative Commons Namensnennung 4.0 International Lizenz veröffentlicht, welche die Nutzung, Vervielfältigung, Bearbeitung, Verbreitung und Wiedergabe in jeglichem Medium und Format erlaubt, sofern Sie den/die ursprünglichen Autor(en) und die Quelle ordnungsgemäß nennen, 
einen Link zur Creative Commons Lizenz beifügen und angeben, ob Änderungen vorgenommen wurden.

Die in diesem Artikel enthaltenen Bilder und sonstiges Drittmaterial unterliegen ebenfalls der genannten Creative Commons Lizenz, sofern sich aus der Abbildungslegende nichts anderes ergibt. Sofern das betreffende Material nicht unter der genannten Creative Commons Lizenz steht und die betreffende Handlung nicht nach gesetzlichen Vorschriften erlaubt ist, ist für die oben aufgeführten Weiterverwendungen des Materials die Einwilligung des jeweiligen Rechteinhabers einzuholen.

Weitere Details zur Lizenz entnehmen Sie bitte der Lizenzinformation auf http://creativecommons.org/licenses/by/4.0/deed.de.

\section{S. https://www.dfb.de/news/detail/kampf-gegen-rassismus-wor- auf-es-nun-ankommt-225555/.}

2. Um Missverständnisse zu vermeiden: Selbstverständlich gibt es auch Gegentendenzen und Widerstände auch innerhalb staatlicher Institutionen, weshalb ist die Reichweite dieser Tendenz differenziert zu betrachten ist.

3. S. https://www.rassismusmonitor.de/.

4. Als instruktiven Überblick dazu s. Kerner (2013).

5. S. dazu u. a. Brubaker (2007, S. 85ff).

6. Eine ausführliche kritische Auseinandersetzung mit dem Rezept kollektiver Identität hat Niethammer (2000) vorgelegt.

7. Um Missverständnisse zu vermeiden: Armin Pfahl-Traughber lehnt eine Gleichsetzung rechter und linker Spielarten von Identitätspolitik explizit ab (2021, S. 151) und ist nicht als Vertreter einer solchen unterkomplexen Extremismustheorie einzuordnen.

8. Dies gilt jedoch selbstverständlich nicht für rechtsextreme Strömungen.

9. Es soll damit keineswegs unterstellt sein, dass alle identitätspolitischen Positionen durch eine solche Vereinfachung gekennzeichnet sind, obwobl ein reflexives Verständnis komplexer, in sich ambivalenter Identitäten wenig für einen identitätspolitischen Aktivismus geeignet ist.

10. Ein wichtiger Ausgangspunkt dafür ist die Position eines schwarzen Nationalismus, die Malcom X in Abgrenzung zu Martin Luther King eingenommen, später aber explizit revidiert hat (s. Jones 2020). Zu Martin Luther King, dessen komplexe Position in Deutschland gewöhnlich nur grob vereinfacht rezipiert wird, hat Albert Scharenberg (2011) eine instruktive Studie vorlegt.

11. S. dazu für die rassismuskritische Bildungsarbeit z. B. Broden (2017).

\section{Literatur}

Amnesty International (2017). Glossar für diskriminierungssensible Sprache. https://www.amnesty.de/2017/3/1/glossar-fuer-diskriminierungssensible-sprache. Zugegriffen: 11. Aug. 2021

Batov, O. (2004). Der alte und der neue Antisemitismus. In D. Rabinov \& U. Speck (Hrsg.), Nener Antisemitismus? Frankfurt: Suhrkamp.

Broden, A. (2017). Rassismuskritische Bildungsarbeit. In K. Fereidooni \& M. El (Hrsg.), Rassismuskritik und Widerstandsformen (S. 819-835). Wiesbaden: Springer.

Brubaker, R. (2007). Ethnizität ohne Gruppen. Hamburg: Hamburger Edition.

Bundesregierung (2016). Strategie der Bundesregierung zur Extremismusprävention und Demokratieförderung. https://www.bmi.bund.de/SharedDocs/downloads/DE/veroeffentlichungen/2016/strategie-extremismuspraevention-und-demokratiefoerderung.html. Zugegriffen: 11. Aug. 2021
DiAngelo, R. (2018a). White fragility: why it's so hard for white people to talk about racism. Boston: Beacon Press.

DiAngelo, R. (2018b). „Die meisten Weißen sehen nur expliziten Rassismus“. Interview in Zeit Campus. https://www.zeit.de/campus/2018-08/rassismus-dekonstruktion-weisssein-privileg-robin-diangelo/komplettansicht. Zugegriffen: 11. Aug. 2021

Dikötter, F. (2018). Wie und warum wurde Rasse zu einem globalen Begriff? In N. Foroutan, et al. (Hrsg.), Das Phantom „Rasse“ (S. 131-150). Bonn: Bundeszentrale für politische Bildung.

Eddo-Lodge, R. (2019). Warum ich nicht länger mit Weißen über die Hautfarbe spreche. Bonn: Bundeszentrale für politische Bildung.

Fereidooni, K. (2020). Über Diskriminierung sprechen. Worte finden. https://www.gew-berlin.de/aktuelles/detailseite/neuigkeiten/worte-finden/. Zugegriffen: 11. Aug. 2021

Funk, M. (2021). Verlust der politischen Heimat. Die Zeit, 10.06.2021.

Gauck, J. (2021). Menschen, die die Freiheit, Demokratie und Menschenrechte lieben, fragen nicht danach, ob jemand schwarz ist oder weiß. Die Zeit, 31.03.2021.

Jones, T. (2020). The ideological and spiritual transformation of Malcolm X. Journal of African American Studies, 24, 417-433. https:// doi.org/10.1007/s12111-020-09487-2.

Kerner, I. (2013). Critical Whiteness Studies: Potentiale und Grenzen eines wissenspolitischen Projekts. Feministische Studien, 31(2), 278-293.

Mayer, M. (2021). Demokratische Mehrheiten in Washington und eine erneuerte US-Linke. Prokla, 51(203), 221-244.

Michels, W. B. (2006). The trouble with diversity. How we learned to love identity and ignore inequality. New York: Holt.

Misk, R. (2019). Die falschen Freunde der einfachen Leute. Frankfurt: Suhrkamp.

Niethammer, L. (2000). Kollektive Identität. Heimliche Quellen einer unheimlichen Konjunktur. Reinbek: Rowohlt.

Pfahl-Traughber, A. (2020). Antiindividualismus und Antiuniversalismus als Konsequenzen. perspektiven ds - Zeitschrift für Gesellschaftsanalyse und Reformpolitik, 37, 137-152.

Pfahl-Traughber, A. (2021). Gefährliche Nähe. Die Zeit, Nr. 12, 18.03.2021.

Raether, T. (2018). Hört auf zu jammern, alte weiße Männer! https://szmagazin.sueddeutsche.de/leben-und-gesellschaft/hoert-auf-zu-jammern-alte-weisse-maenner-85975. SZ Magazin, 09.08.2021. Zugegriffen: 11. Aug. 2021

Scharenberg, A. (2011). Martin Luther King: Ein biografisches Porträt. Freiburg: Herder.

Scherr, A. (2017). Rassismus, Post-Rassismus und Nationalismus. Erfordernisse einer differenzierten Kritik. Peripherie, 37, 233-249.

Steinert, H. (1998). Genau hinsehen, geduldig nachdenken und sich nicht dumm machen lassen. In Ders. (Hrsg.), Zur Kritik der empirischen Sozialforschung. Ein Methodengrundkurs. Studientexte zur Sozialwissenschaft, (Bd. 14, S. 67-79). Frankfurt/Main: Fachbereich Gesellschaftswissenschaften, Goethe Universität.

Vranicki, P. (1974). Geschichte des Marxismus. Bd. 2. Frankfurt: Suhrkamp.

Wagenknecht, S. (2021). Die Selbstgerechten: Mein Gegenprogramm - für Gemeinsinn und Zusammenhalt. Frankfurt/New York: Campus.

\section{Weiterführende Literatur}

Corbman, M. (2020). The creation of the devil and the end of the white man's rule: the theological influence of the Nation of Islam on early black theology. Religions, 11, 1-14. https://www.mdpi.com/2077-1444/11/6/305.

King, M. L. (1957). The birth of a new nation. https://kinginstitute.stanford.edu/king-papers/documents/birth-new-nation-sermon-delivered-dexter-avenue-baptist-church. Zugegriffen: 11. Aug. 2021 